\title{
Comparison of the magnetic equivalent convection direction and ionospheric convection observed by the SuperDARN radars
}

\author{
L. V. Benkevitch ${ }^{1}$, A. V. Koustov ${ }^{1}$, J. Liang ${ }^{2}$, and J. F. Watermann ${ }^{3}$ \\ ${ }^{1}$ Institute of Space and Atmospheric Studies, University of Saskatchewan, 116 Science Place, Saskatoon, S7N 5E2 Canada \\ ${ }^{2}$ Canadian Space Agency, Saint-Hubert, Quebec, Canada \\ ${ }^{3}$ Danish Meteorological Institute, Lyngbyvej 100, 2100 Copenhagen, Denmark
}

Received: 23 May 2006 - Revised: 29 August 2006 - Accepted: 20 September 2006 - Published: 21 November 2006

\begin{abstract}
SuperDARN radar and high-latitude magnetometer observations are used to statistically investigate quality of the convection direction estimates from magnetometer data if assumption is made that the magnetic equivalent convection vector (MEC) corresponds to the convection direction. The statistics includes five full days, $\sim 75000$ of joint individual measurements for different seasons. It is demonstrated that the best (worst) agreement between the MEC and ionospheric convection occurs for the sunlit, summer (dark, winter) ionosphere. Overall, the MEC direction is reasonable (deviates less than $45^{\circ}$ from the SuperDARN direction) in at least $\sim 55 \%$ of points and it is better for the latitudes of the auroral oval. In terms of the magnetic local time, the agreement is the best (worst) in the dusk (early morning) sector. Possible reasons for differences between the MEC and ionospheric convection directions are discussed.
\end{abstract}

Keywords. Ionosphere (Auroral ionosphere; Ionospheric irregularities; Plasma convection)

\section{Introduction}

Ground-based magnetometer observations have been providing invaluable information that allows physicists to study various processes occurring in the near Earth space (e.g., Kamide, 1988; Kivelson and Russell, 1995). One of such processes is global plasma circulation in the Earth's ionosphere-magnetosphere cavity. Over the years, several methods have been advanced to infer the plasma flow parameters from ground-based magnetometer observations (Kamide, 1988). One of the most advanced methods, the AMIE technique, combines ground magnetic measurements with other data such as ion drift measurements on satel-

Correspondence to: A. V. Koustov

(sasha.koustov@usask.ca) lites and coherent/incoherent radar data on the electric field (Richmond and Kamide, 1988). Auroral images from satellites can also, in principle, be included to obtain the conductance estimates. Despite the fact that the AMIE method and alike are gaining more and more popularity, they are not always readily accessible. The plasma convection direction and relative intensity are still often estimated by relating it to the magnetic equivalent convection (MEC), the vector obtained by simple rotation of the horizontal magnetic perturbation vector anticlockwise by $90^{\circ}$. The validity of such an estimate is based on the assumption that the magnetic perturbation detected on the ground is entirely determined by the ionospheric Hall currents. If one assumes that the conductance distribution in the ionosphere is spatially uniform, then the above method of convection velocity estimates from ground-based magnetometer data is justifiable by the so called Fukushima's theorem (Fukushima, 1976) stating that the magnetic effect on the ground due to the vertical fieldaligned current is cancelled by the magnetic effect due to the Pedersen current (flowing perpendicular to the magnetic field along the electric field direction). Generally, the condition of uniformity of the high-latitude ionospheric conductance cannot be accepted but it is often argued that errors in convection estimates using MEC approach are insignificant, especially for the sunlit summer ionosphere where spatial variations of the conductance are not significant. Clearly, the validity of this approach is an issue for every specific event.

Lyatsky and Maltsev (1982) theoretically predicted significant differences between the MEC and real convection directions for observations near the solar terminator where the ionospheric conductance experiences a drastic change. These analytical estimates were confirmed by a numerical solution of appropriate equations of high-latitude electrodynamics by Benkevitch and Lyatsky (2000). Earlier modeling by Zhu et al. (1997) suggested strong MEC and true convection differences for observations of traveling convection vortices, excited by a pair of field-aligned current

Published by Copernicus GmbH on behalf of the European Geosciences Union. 


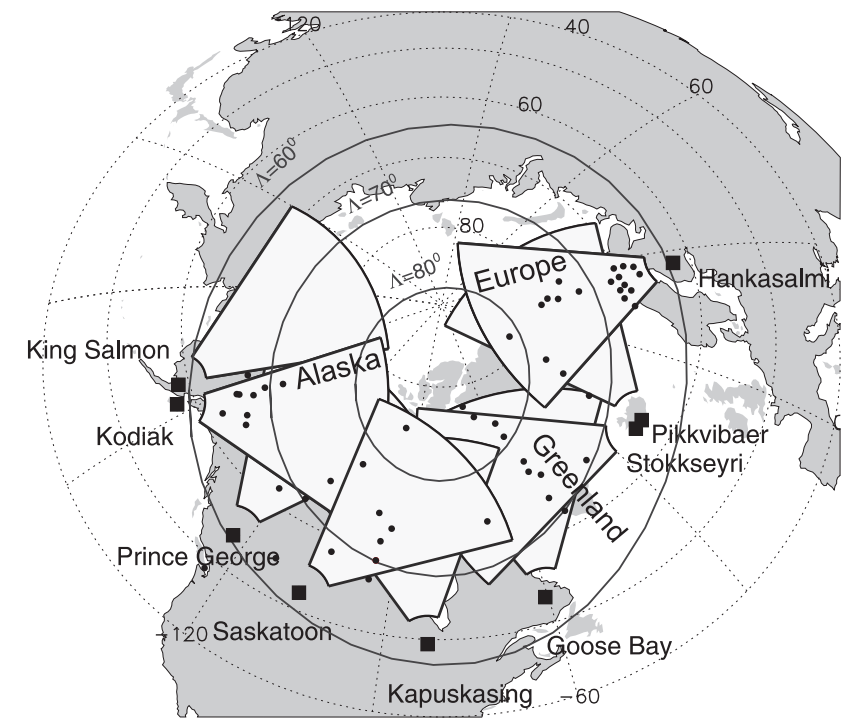

Fig. 1. A map showing the Northern hemisphere SuperDARN radar locations (bold squares), their FoVs (circular sectors), and the magnetometer locations (solid dots). Actual magnetometer data in these sectors (Alaska, Greenland, and Northern Europe) will be presented.

filaments quickly moving in the azimuthal direction on the dayside. Lyatsky et al. (2000) pointed out that strong interhemispheric currents can flow between the hemispheres; this effect can complicate the relationship between the MEC and true convection directions as well. The above theoretical studies indicate that the magnetic effect of field-aligned currents on the ground might not be often compensated by the magnetic effect of the Pedersen currents, especially if a magnetometer monitors magnetic effect of remote currents.

On the experimental side, one of the ways to test whether the magnetic effects of field-aligned and Pedersen currents are compensated is to look at the data on local ionospheric conductance, electric field and magnetic perturbations. Observations showed that, for some periods, the agreement between the expected magnetic perturbations and the measured ones seems to be reasonable while for others it is poor (e.g., Brekke et al., 1974). So far, only a few papers have been published in which the MEC and ionospheric convection were directly compared (e.g., Lyatsky et al., 1999; Huang et al., 2000). Lyatsky et al. (1999) investigated several vortex events detected by the SuperDARN radars and their associated magnetic equivalent currents and showed that the MEC patterns were displaced equatorward with respect to the SuperDARN convection patterns. For the dayside winter ionosphere this shift was attributed to the effect of the conductance gradient near the ionospheric projection of the solar terminator line. Huang et al. (2000) studied the agreement between the magnetometer- and SuperDARN-inferred convection vortices for various MLT sectors. They showed that the patterns have rather good agreement between 12:00-
15:00 MLT, and the agreement worsens around 18:00 MLT. This fact was also explained by the presence of large conductance gradients near the day-night transition region.

The above studies utilized only a small number of individual magnetometers to compare with convection data. The goal of this paper is to give a more comprehensive, quantitative assessment of the difference between the MEC and the ionospheric convection direction inferred from concurrent SuperDARN radar measurements.

\section{Approach to the comparison}

The SuperDARN HF radars are designed to monitor plasma convection in the Northern and Southern high-latitude ionospheres (Greenwald et al., 1995). In the Northern hemisphere, the radars cover significant portion of the ionosphere with field of views (FoVs) of individual radars stretching from Northern Europe to Alaska, Fig. 1. The global convection maps are produced every one or two minutes using fit potential technique of Ruohonimei and Baker (1998).

Northern hemisphere magnetometers are located in four distinct sectors, Northern Europe, Greenland, Northern Canada and Alaska, Fig. 1. They are distributed all across FoVs of individual SuperDARN radars with somewhat better coverage at latitudes of the auroral oval $\left(\sim 70^{\circ}\right.$ MLAT). Magnetometer measurements have better temporal resolution, ranging from $1 \mathrm{~s}$ to $20 \mathrm{~s}$. In this study, the MEC was calculated by considering the magnetic perturbations in the north-south and east-west components with respect to the baselines selected as the daily averages for five quietest days around the event considered. We should note that analysis was also performed by considering the daily mean baselines (Benkevitch, 2006), and the results obtained are not significantly different from what is reported in the present study.

According to Fig. 1, radar and magnetometer data can be compared in about 50 locations though in practice joint data are only available in about half of the magnetometers because HF echoes are typically observed only in fractions of the radars' FoVs. In this study, five days in 2001 were selected for the analysis. These are 25 January, 9 February, 20 March, 15 July, and 13 December. These days were selected for a simple reason that each individual SuperDARN convection map contained several hundred points all day long so that the radar and magnetometer data were possible to compare in 20-25 points for every SuperDARN convection map.

Figure $2 \mathrm{a}$ is an example of the SuperDARN convection map presented as contours of the electrostatic potential in magnetic latitude (MLAT) - magnetic longitude (MLON) coordinates. The ACCGM coordinates were used. Overlaid on the map are the MEC vectors obtained for the same period of time (the closest to the middle of the 1-min sweep period) and the locations of SuperDARN echoes, plus signs. Presenting SuperDARN data in the form of potential contours is convenient for the present work as the plasma flow goes 


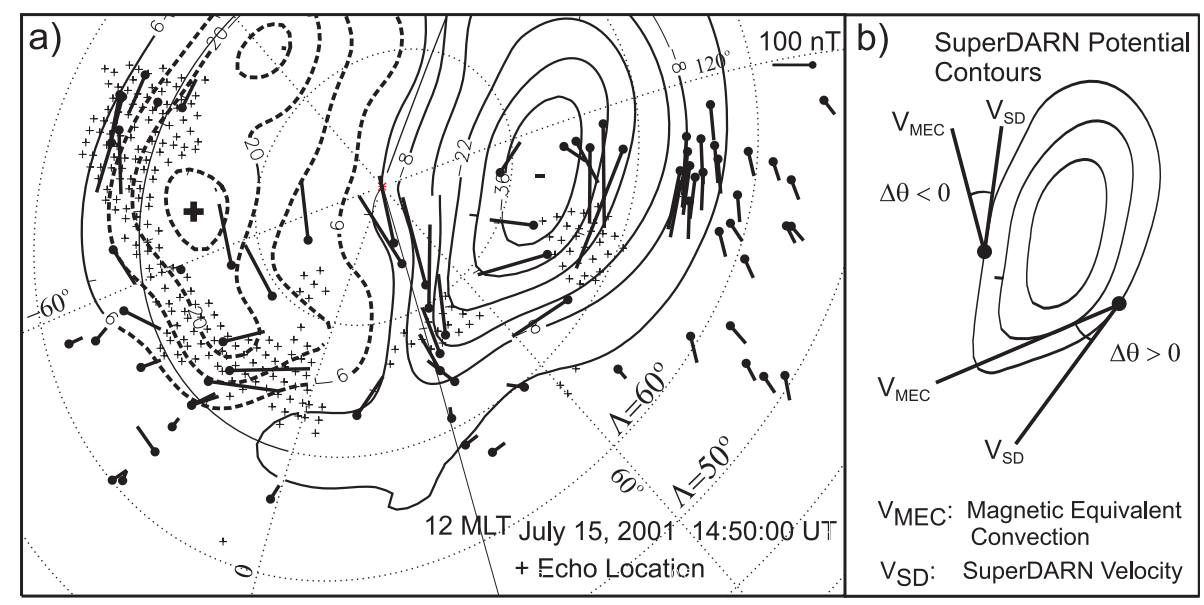

Fig. 2. (a) SuperDARN contour map of the electrostatic potential (in $\mathrm{kV}$ ) and the equivalent convection vectors (lines with large dots at their origin) expressed in nT. The magnetic (ACCGM) coordinate system is used (dotted lines). The "+" sign indicates the radar echo location. (b) A scheme illustrating two possible mutual orientations of the convection (SuperDARN, $\mathrm{V}_{S D}$ ) and magnetic equivalent convection vector $\mathrm{V}_{\mathrm{MEC}}$ and assigned signs for the azimuth difference angle $\Delta \theta$. Notice that the $\mathrm{V}_{S D}$ vector at any point is tangential to the potential contour line.

along the lines of equal potential so that the difference between the magnetometer and radar directions of the plasma flow can be calculated overhead of every magnetometer location. In Fig. 2a the solid (dashed) contour corresponds to the $\mathrm{CW}(\mathrm{CCW})$ direction of the plasma flow. We measure the MEC magnitude in nano-Teslas; the appropriate scale is shown in the upper right corner.

One can notice in Fig. 2a that for some MEC vectors there are no radar echoes in their vicinity. Such points were not considered for a radar-magnetometer comparison because the radar convection estimates (the shape of a potential contour) are not reliable in these parts of the ionosphere. We included into statistics the MEC data from only those magnetometers that had at least one radar measurement within a circular vicinity of a station of the radius of $160 \mathrm{~km}$ (roughly, the azimuthal size of a radar cell).

In Fig. 2a, for the areas with good radar coverage, the MEC vectors are aligned with the SuperDARN convection direction reasonably well. There are some differences; the MEC vectors deviate either clockwise or counter-clockwise. We characterise the MEC-ionospheric convection direction difference $\Delta \theta$ by a positive (negative) angle if the MEC vector is rotated $\mathrm{CW}(\mathrm{CCW})$ from the direction of the potential contour, Fig. 2b. We imposed additional restrictions on the data that we included into the statistics, namely we considered only those radar (magnetometer) measurements for which the radar velocity (magnetic perturbation) was over $100 \mathrm{~m} / \mathrm{s}$ (>5 nT). These restrictions were introduced to exclude potential effects of ground scatter contribution to the radar velocities (effectively reducing it) and uncertainty in the baseline determination for the magnetometers.

\section{Comparison for various solar illumination conditions}

Previous publications (e.g., Lyatsky et al., 1999) suggested that variations of the ionospheric conductance produced by solar illumination of the high-latitude ionosphere affect the relationship between the MEC and convection. In this section we attempt to asses the role of solar illumination by considering one full day of joint radar-magnetometer observations and sorting out obtained data according to the illumination conditions. We selected the day of 9 February 2001, near vernal equinox. For this day, SuperDARN was providing $\sim 700$ vectors for each 1-min convection map (on average) so that reasonable data statistics is available for various illumination conditions. We distinguish observations in the sunlit, transition and dark ionospheres by assigning the solar zenith angle $\chi$ to each individual point of a comparison as $\chi<87^{\circ}, 87^{\circ}<\chi<93^{\circ}$, and $\chi>93^{\circ}$, respectively.

In terms of the magnetic activity, 9 February 2001 was modestly disturbed day; the planetary $K p$ index was between 1 and 2 with an average value of $\sim 1.5$. To give better perception as to what kind of magnetic activity was observed on this day, we present in Fig. 3 magnetic perturbations in the north-south component of the magnetic field from three networks GIMA (Alaska), DMI (Greenland), and IMAGE (Northern Europe). The magnetometers for each of these networks are located along about the same magnetic meridian, and the three meridians cover almost entirely the combined FoV of the SuperDARN radars, Fig. 1. One can see, Fig. 3, that maximum observed perturbations were of the order of $200 \mathrm{nT}$; these values were lasting for less than $2 \mathrm{~h}$ in each sector. Finally, in terms of the IMF orientation in the Y-Z plane, there were no preferential conditions, implying that the performed comparison is independent of the IMF orientation. 


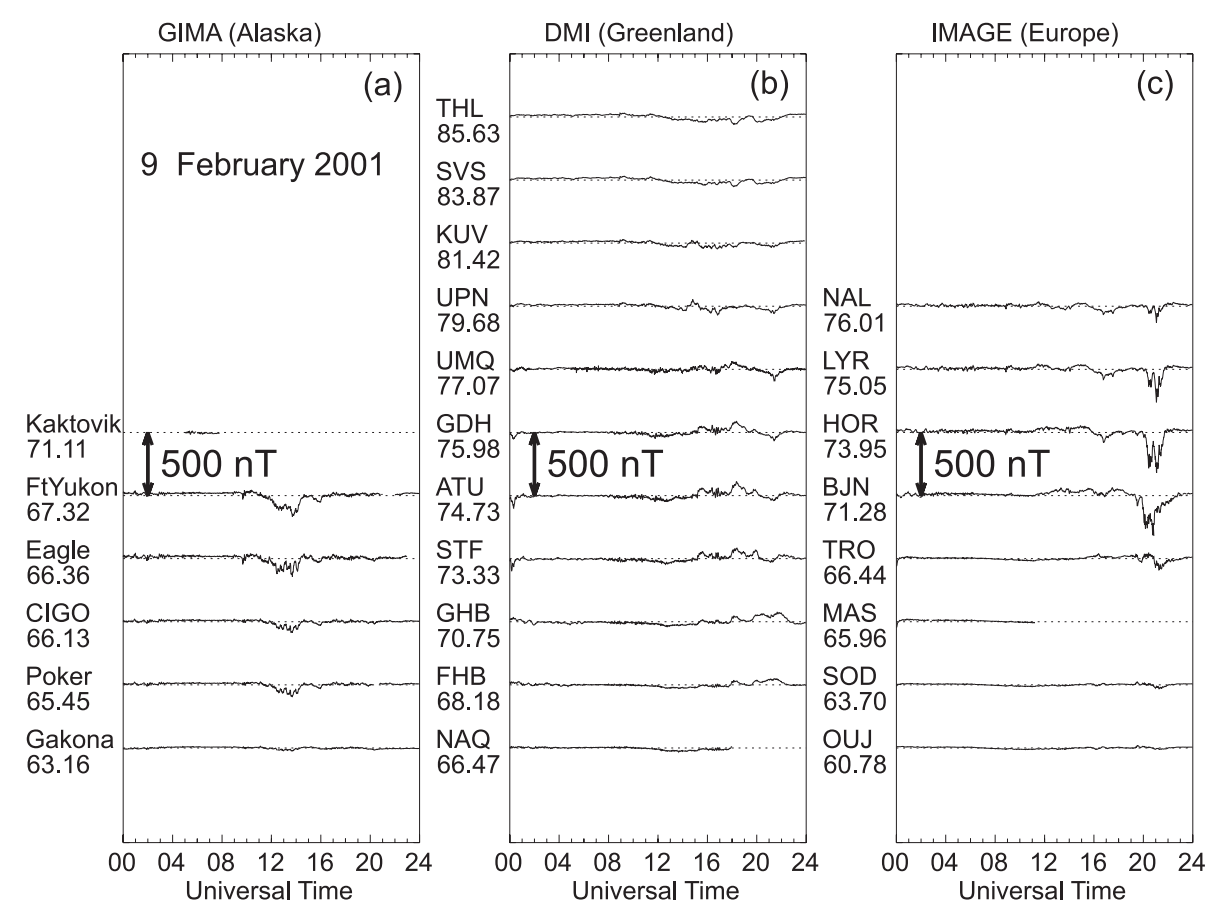

Fig. 3. Stackplots of North-South magnetic field component perturbations for 9 February 2001 in (a) Alaskan, (b) Greenland, and (c) European sectors. The names of magnetometer stations and respective AACGM latitudes are given on the left hand side of each plot.

Figure 4 presents the results of the comparison. Here we show three histogram plots for the angle $\Delta \theta$ at three different illumination conditions, sunlit, transition and dark ionospheres. In each diagram, the occurrence rates are normalized to one. In the upper right corner of each panel we present the total number of available points and the number of points corresponding to the maximum of the distribution. Also shown are the mean value of the distribution, the standard deviation from the mean (width of the distribution) and the number of points for which the radar-magnetometer directions differ by less than $45^{\circ}$ (we shall classify these points as the points of reasonable/good agreement).

Assessing the diagrams, one can say that all three distributions have one major peak with significant "tails" stretching to large values of $\Delta \theta$, up to $\pm 180^{\circ}$ (the MEC is opposite to the ionospheric convection). The distributions are fairly symmetric except the data for the transition ionosphere, which demonstrate a secondary maximum around $\Delta \theta=+60^{\circ}$. The mean values for the distributions are around zero indicating an overall tendency for the MEC direction to be close to the ionospheric convection. In terms of the distribution width, the sunlit comparison shows the narrowest one. For the majority of points in all the three diagrams the direction difference $\Delta \theta$ is less than $\pm 45^{\circ}$. To ease visual evaluation of this number, we introduced different coloring in all histograms; bins with $\Delta \theta$ within $\pm 45^{\circ}$ have lighter shading. One can see that there are $65 \%, 57 \%$, and $47 \%$ of good points for the dayside, transition and dark ionospheres.
In attempt to investigate any seasonal effects in the difference between the MEC and ionospheric convection, we selected two additional events, one for winter (13 December) and one for summer (15 July). For the 15 July (13 December) event data were mostly available in the sunlit (dark) ionosphere, as expected. For both events, the magnetic activity was not strong, $K p$ indices were between 1 and 2 . For the summer event, the IMF Bz component was preferentially negative while for the winter event all IMF orientations in the Y-Z plane were available (actual data can be found in the thesis work by Benkevitch, 2006).

Results of the comparison are presented in Fig. 5. One can see that both distributions are quite symmetric with stronger tails (broader distribution) for the winter event. The mean values of the distributions are around zero, though again the summer event shows less departure from zero. The majority of points, $71 \%$ for summer and $56 \%$ for winter, shows the direction difference less than $45^{\circ}$.

\section{Comparison for various magnetic latitudes and MLT sectors}

Now we investigate the relationship between the MEC direction and ionospheric convection at various latitudes and in various magnetic local time sectors. We expect the magnetic latitude effect because even for relatively quiet conditions, conductance is enhanced at latitudes of the auroral oval thus giving at least a latitudinal gradient in the conductance. 


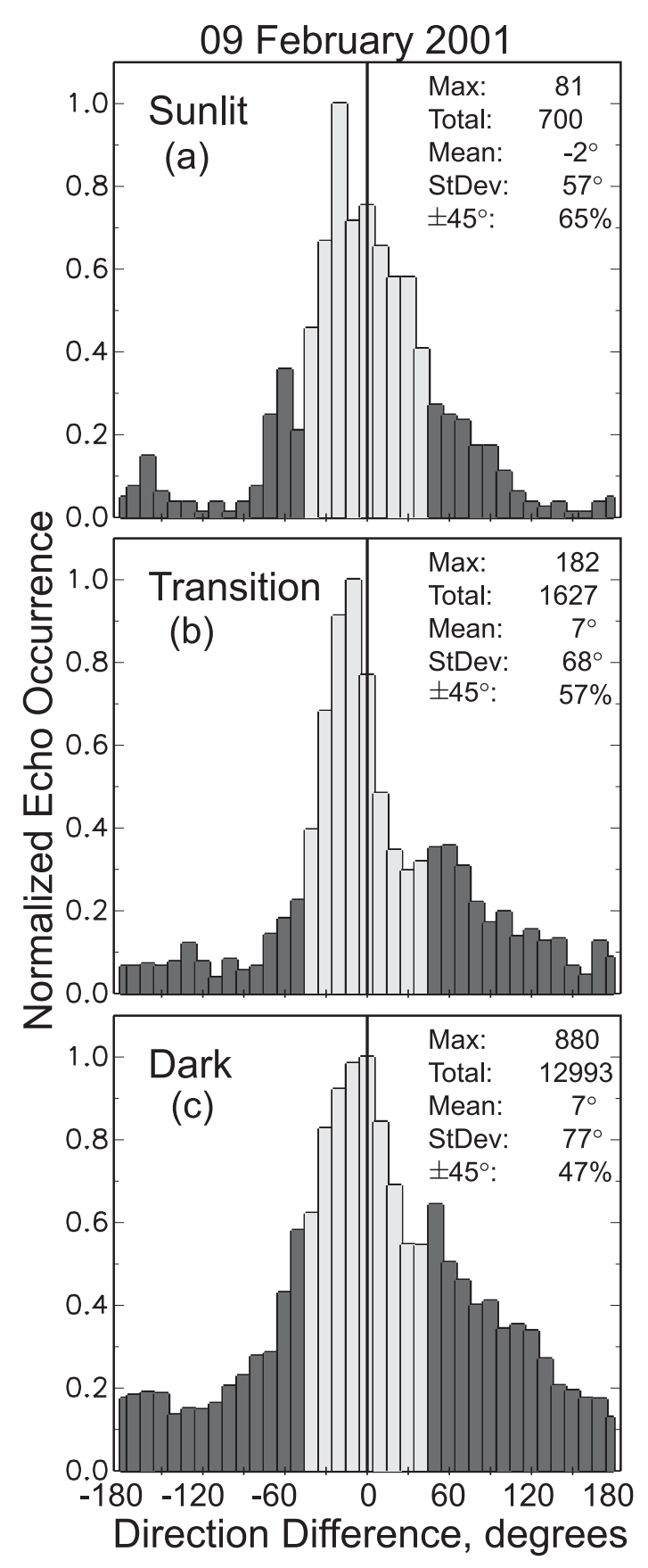

Fig. 4. Statistical distributions for the angle $\Delta \theta$ (the azimuth difference between the SuperDARN velocity and the magnetic equivalent convection vectors) on 9 February 2001 (near vernal equinox). The comparison is performed for the (a) sunlit, (b) transition, and (c) dark ionospheric regions. $10^{\circ}$ bins of $\Delta \theta$ are used. The histograms are normalized to one. Also shown are the number of points corresponding to the bin of the distribution maximum, total number of points available, the mean value of the distribution (in degrees), the standard deviation of the distribution (in degrees), and the percentage of data points with $\Delta \theta$ within $\pm 45^{\circ}$.

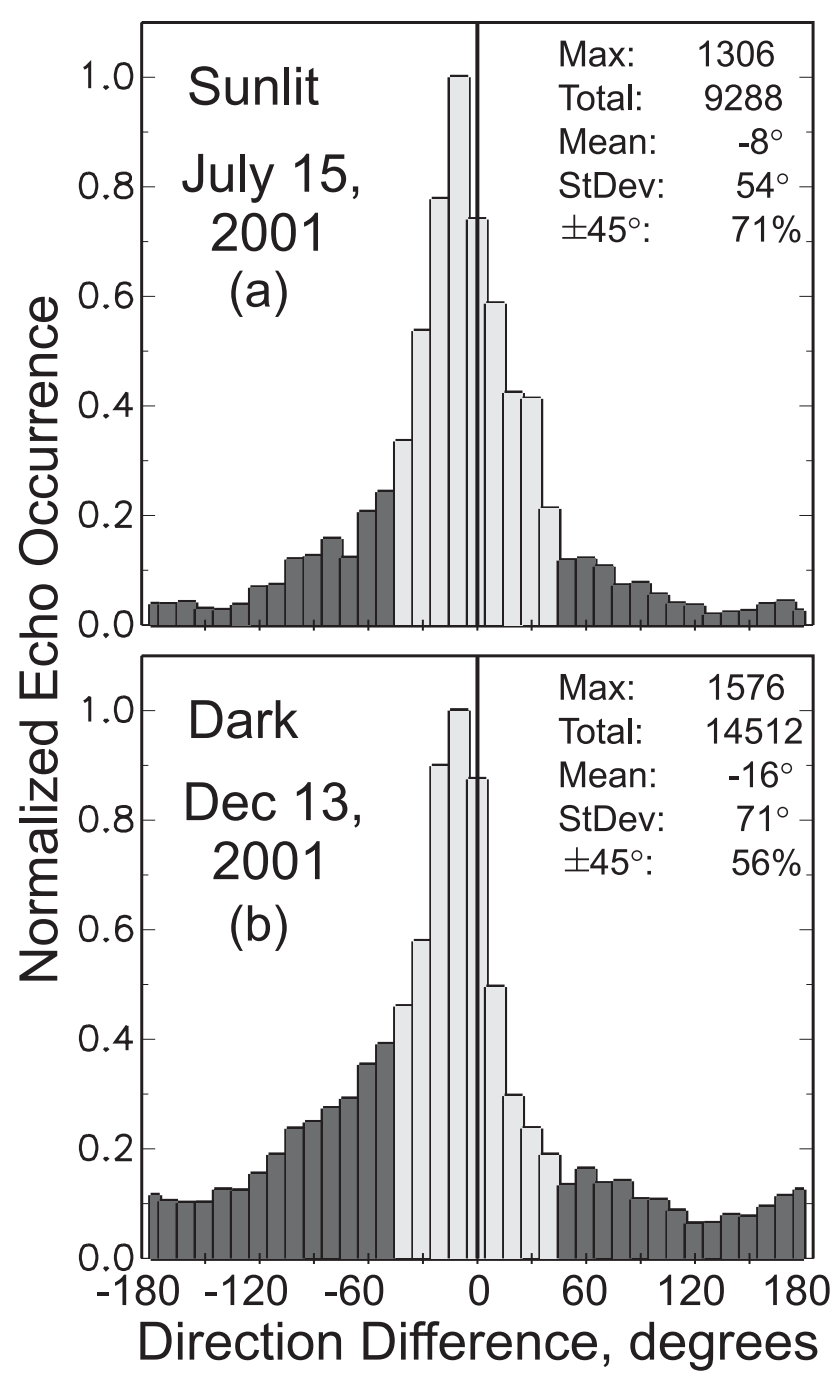

Fig. 5. The same as in Fig. 4 but for 15 July 2001 and 13 December 2001.

We also expect the magnetic local time effects because, for example, the statistically inferred configuration and intensity of Region 1 and Region 2 field-aligned currents (affecting magnetometer response) change in different MLT sectors.

To increase statistics, we consider now data for five days, the three days considered in the previous section, and two other ones, 20 March and 25 January. There should not be a concern for merging the data from different seasons since we illustrated that histograms of $\Delta \theta$ for various seasons do not differ drastically.

We first sort the data according to the MLT sector, without consideration of the magnetic latitude variation. To accomplish this, we split the entire available data set into four individual sets corresponding to four MLT sectors: dayside (09:00-15:00 MLT), dusk (15:00-21:00 MLT), dawn (03:00-09:00 MLT) and nightside (21:00-03:00 MLT). Such a consideration provides an opportunity to compare the MEC 


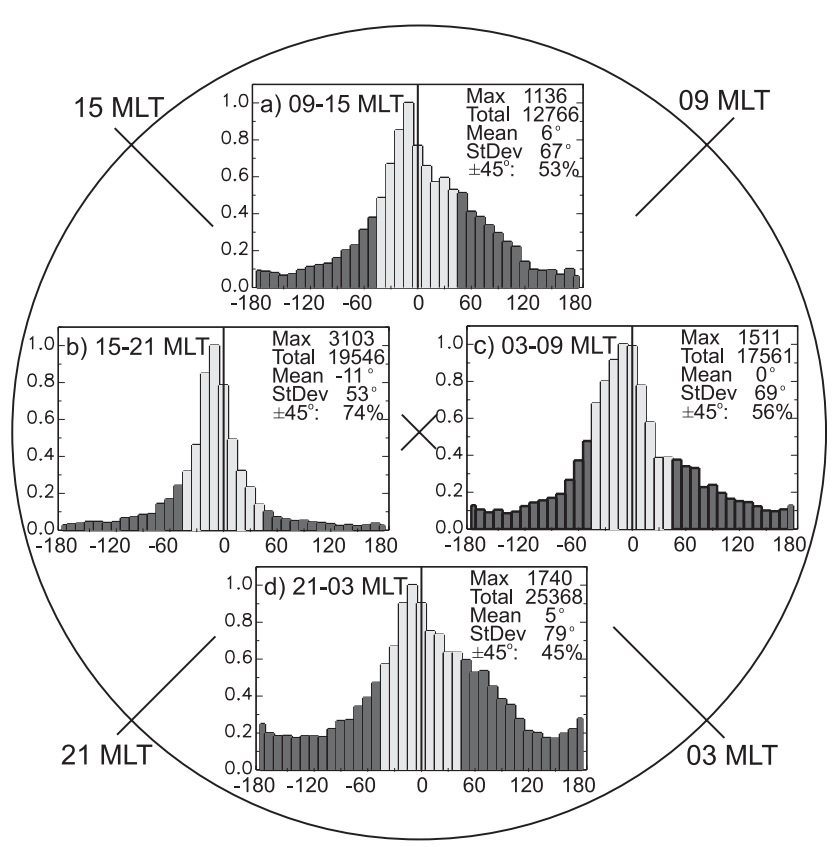

Fig. 6. Statistical distributions for the angle $\Delta \theta$ in four magnetic local time sectors: (a) dayside (09:00-15:00 MLT), (b) dusk (15:00-21:00 MLT), (c) dawn (03:00-09:00 MLT) and (d) nightside (21:00-03:00 MLT). All distributions were normalized to one. Also shown are the number of points corresponding to the bin of the distribution maximum, total number of points available, the mean value of the distribution (in degrees), the standard deviation of the distribution (in degrees), and the percentage of data points with $\Delta \theta$ within $\pm 45^{\circ}$. Data for five days were used.

direction-convection relationship in distinctly different MLT sectors where response of the high-latitude ionosphere to external drivers can be quite different. In addition, for such a comparison there is significant number of measurements and statistics is comparable for all sectors, with total number of measurements around 20000 in each sector.

Figure 6 shows that in all four sectors the distributions for $\Delta \theta$ are more or less symmetric with the maximum shifted from zero by several degrees. The distribution for the duskside shows largest mean deviation from zero, negative $7^{\circ}$. On the other hand, this distribution has very little skewness and smallest tails so that its width is smallest out of four cases. In this sector, remarkable $74 \%$ of points exhibit good agreement of MEC direction and ionospheric convection. The distribution for the nightside has strongest tails and the number of points of good agreement is less than $50 \%$. The asymmetry of the distribution is introduced by a somewhat larger number of points with $\Delta \theta=30^{\circ}-60^{\circ}$. This gives an overall shift of the distribution towards positive $\Delta \theta$ though the maximum in the distribution is achieved at negative $\Delta \theta$. We note that similar distribution is observed for the dayside measurements. In the morning sector, the skewness of the distribution is owing to somewhat increased amount of points with negative
$\Delta \theta \sim-30^{\circ}$.

On our next step, we consider the $\Delta \theta$ distributions for various magnetic latitudes and MLT sectors. To accomplish this, the entire available magnetometer-radar data set was partitioned according to eight MLT sectors and $5^{\circ}$ magnetic latitude gates within each sector. The criterion for such data segmentation was that there would be reasonable amount of measurements for each segment. To simplify the results overview, instead of considering all histograms similar to the ones shown in Figs. 4-6, we present information on the mean value, standard deviation, and percentage of the data points with $\Delta \theta$ within $\pm 45^{\circ}$, Fig. 7 .

Figures $7 \mathrm{a}, \mathrm{b}$ show the data for the mean shifts of the histograms. One can see that the worst agreement (up to $+60^{\circ}$ ) is at very high latitudes of $>80^{\circ}$. Strong disagreements of $>30^{\circ}$ at these latitudes occur between 00:00 and 18:00 MLT. One can also notice strong disagreements at low latitudes, in the sectors of 09:00-15:00 MLT and 21:0000:00 MLT. Somewhat better, but still not very good agreement of $20^{\circ}<\Delta \theta<30^{\circ}$, is observed in the sectors of 15:0021:00 MLT and 00:00-03:00 MLT. Data in the above sectors indicate that the overall agreement at low latitudes is rather poor. The agreement seems to be better at intermediate magnetic latitudes of $65^{\circ}-75^{\circ}$ (typical latitudes of the auroral oval) as compared to the low and high latitudes. Indeed, between 03:00 and 15:00 MLT and in the sector 21:0000:00 MLT, $\Delta \theta$ mean values are less than $20^{\circ}$ and they are better than at other latitudes. In the sectors 18:00-21:00 MLT and 00:00-03:00 MLT, better agreement at latitudes $65^{\circ}$ $75^{\circ}$ is not very obvious; it is seen only on the equatorward (18:00-21:00 MLT) or poleward sides (00:00-03:00 MLT) of the band. Interestingly, the mean $\Delta \theta$ is mostly negative in the 15:00-06:00 MLT sector and mostly positive in the 06:0013:00 MLT sector, in agreement with the data presented in Fig. 6.

In terms of the distribution widths, Fig. 7c, the dayside data show overall smaller values, especially at low latitudes. The broadest distributions are at all latitudes in the 00:0006:00 MLT sector (with exception of very low magnetic latitudes of $60^{\circ}-65^{\circ}$ ) and between $70^{\circ}$ and $85^{\circ}$ on the dayside, between 09:00 and 15:00 MLT.

The above conclusions on the width of the distributions are in agreement with the data presented in Fig. $7 \mathrm{~d}$ where we show the percentage of points for which the MECionospheric convection difference is within $\pm 45^{\circ}$ from the ionospheric convection. The smallest amount of points can be seen at high latitudes and in the early morning sector. Additionally, the amount of points is below $60 \%$ for the pre midnight sector of 21:00-00:00 MLT.

\section{Summary and conclusions}

In this study we compared the MEC direction with the direction of ionospheric convection as measured by the 

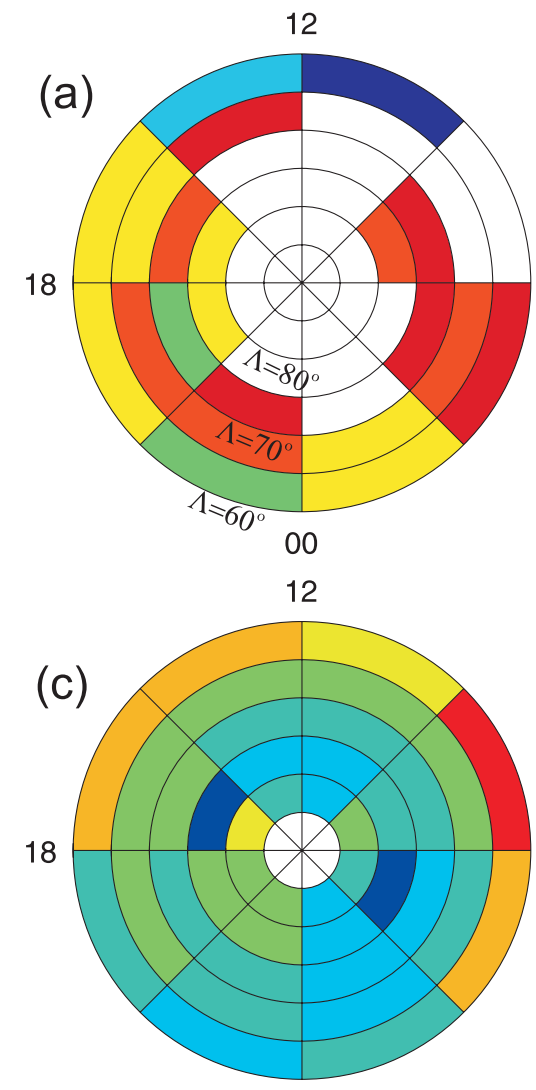

00

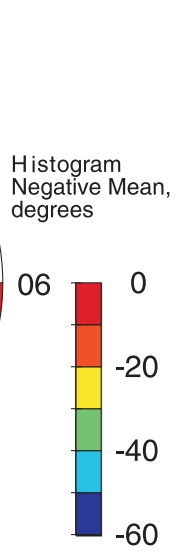

Standard Deviation,

Toc

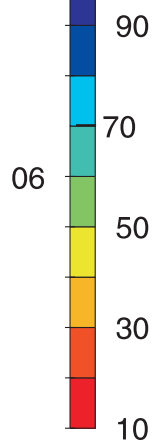

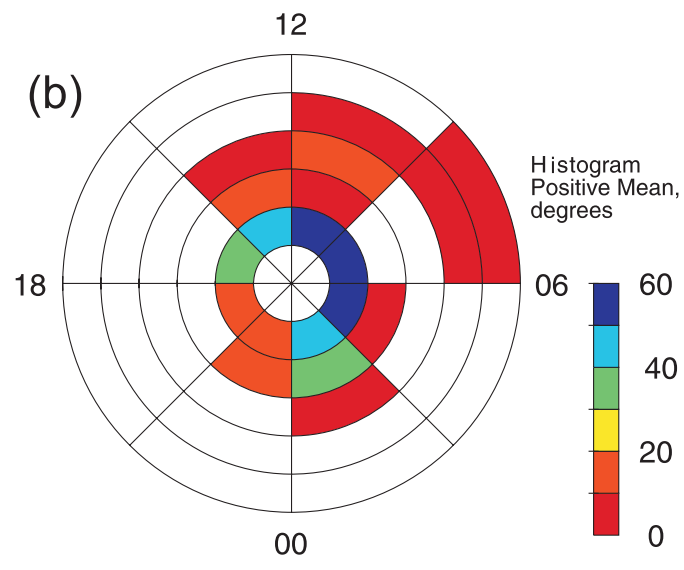

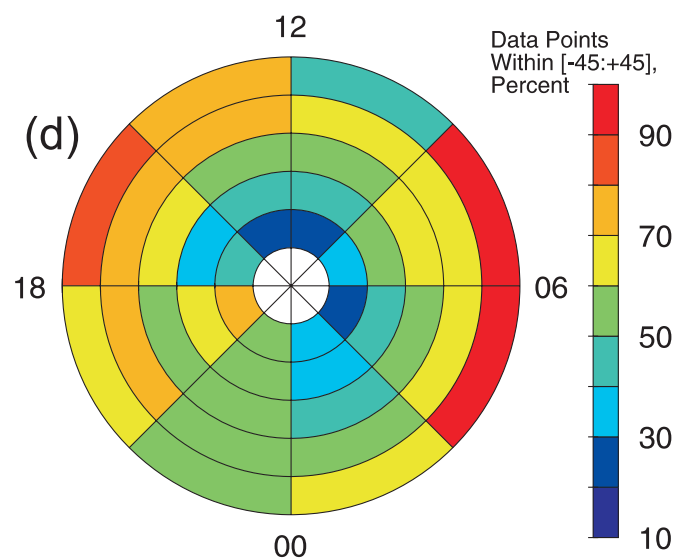

Fig. 7. Color segment diagrams showing parameters of histogram distributions in eight magnetic local time sectors for $5^{\circ}$ steps in the ACCGM latitude (between $60^{\circ}$ and $85^{\circ}$ ): (a) The mean value for negative differences $\Delta \theta$, (b) mean value for positive differences $\Delta \theta$, (c) standard deviation for the angle $\Delta \theta$ and (d) the percentage of points with $\Delta \theta$ within $\pm 45^{\circ}$. Data for five days in various seasons of 2001 were used.

SuperDARN radars with a goal to obtain quantitative sense as to how large are the differences and at what conditions they occur. First we sorted the available data according to the solar illumination conditions and then according to the magnetic latitude and magnetic local time sector of observations.

Performed magnetometer-radar comparison for the equinox event (for which reasonable amount of data was available for the sunlit, dark and transition ionospheres) showed that overall the degree of agreement is the best for the sunlit ionosphere, as expected. For this condition, all three parameters characterizing the histograms of direction difference $\Delta \theta$ (the mean shift, the width and the percentage of the points of reasonable agreement) are the best. In addition, the distribution is more symmetric. These conclusions are in line with the data for two additional events, one for summer, sunlit and one for winter, dark ionospheres. Both additional events show that with the data statistics increase (up to $\sim 10000-15000$ individual points of a comparison), the distributions show somewhat better clustering around zero though the mean shift of the distribution can slightly increase. For the points of reasonable agreement, negative values of $\Delta \theta$ are more frequent. We should mention that although the mean shift of the distribution for the sunlit ionosphere is negative, the distribution can be skewed with significant amount of points having positive $\Delta \theta$, Fig. $6 \mathrm{a}$.

The surprising result of the comparison is the fact that the mean distribution shifts for all three conditions and three different seasons are close to zero indicating that the MEC gives a reasonable estimate of the convection direction most of the time, irrespective of the solar illumination conditions. This conclusion contrasts with the general expectation that MEC/convection difference is small only for the sunlit summer ionosphere whose horizontal uniformity is the best. We should keep in mind, however, that the events considered in this study were for not strong magnetic disturbances and this was very likely one of the reasons for good agreement. On the other hand, the results might imply that other factors, not so much as the Sun-related gradient of the conductance, influence the MEC-convection differences. One should also keep in mind the fact that the SuperDARN FIT technique smoothes out many local variations of the convection so that 
the radar measurements are highly averaged; a magnetomer also spatially averages.

Benkevitch (2006) investigated potential contribution of the regular conductance gradient, produced by the solar illumination, on the quality of convection direction estimates from magnetometer data. He showed that consideration of the effect improves the estimates but not drastically. For example, in a case of 9 February 2001 event, the number of points of good agreement increased from $57 \%$ to $60 \%$. It seems that the consideration of the effect leads to slightly more symmetric distribution of $\Delta \theta$.

The performed analysis in terms of the MLT and magnetic longitude effects revealed several interesting features. First of all, in terms of the MLT sector, the best agreement of the MEC direction and the ionospheric convection was found in the dusk/evening sector where up to $\sim 75 \%$ of points showed good agreement, Fig. 6 . The worst agreement was found for the midnight sector. For the midnight sector, the distribution was also broadest and quite asymmetric.

More detailed data consideration in terms of magnetic latitude showed that for the observations between $65^{\circ}$ and $75^{\circ}$, typical latitudes for the auroral electrojet, the histogram shifts are relatively small (there is more red-related color at these latitudes as compared to the high-latitude ones, Figs. 7a, b) indicating better radar/magnetometer agreement at these latitudes. One exception is observations in the 18:00-21:00 MLT sector at latitudes between $70^{\circ}$ and $75^{\circ}$ MLAT (differences were as high as $40^{\circ}$ ). Better agreement between the radar and magnetometer data in the auroral zone is also seen in Fig. 7d as more yellow-green color and not so much of blue color of poor agreement.

If one compares observations at magnetic latitudes above $65^{\circ}$ in the pre-midnight sector of 18:00-00:00 MLT and after it, 00:00-06:00 MLT, one can notice two general trends: 1) the distributions are much broader after midnight (Fig. 7c) and 2) the percentage of points with good agreement drops down after midnight (Fig. 7d). These trends are consistent with the notion that different factors control the electrojets intensity in the early-evening and late-morning sectors as suggested by Kamide and Kokubun (1996). In the evening sector, the conductance is usually small while the electric field is strong. While both electric field and conductance contribute to the eastward electrojet intensity, the electric field variations are relatively more important than the conductance variations. This leads to a statistically better agreement between the MEC direction and ionospheric convection. In the region of the Harang discontinuity, around midnight, a switch from the "electric field dominating" to the "conductance dominating" electrojet occurs. In the region of the westward electrojet, the southward electric field is relatively weak while the conductance is large and highly variable in space. This means that the electrojet intensity is strongly controlled by the conductance variations. Importantly, the gradients of the conductance play a significant role in the formation of magnetic perturbations. This would lead to poor agreement be- tween the MEC direction and ionospheric convection around the Harang discontinuity region. The disagreement is expected to be stronger for the periods of substorms during which the ionospheric conductance is highly variable and inhomogeneous. It is not a surprise that the agreement was worst in the early morning sector, a typical area of substormrelated precipitations. We should note that there were not two many substorms for the days considered in this study.

We also noticed that the MEC/ionospheric convection agreement was particularly poor for observations at very high (exception is the dusk sector) and very low magnetic latitudes (in many sectors). We think that instrumental effects can contribute to these disagreements. On the radar side, one should keep in mind that the SuperDARN radars are optimized for convection monitoring at latitudes typical for the auroral oval. At both short and large radar ranges (low and high magnetic latitudes), the radar velocity can be affected by the E region echo contamination (Milan et al., 1997; Lacroix and Moorcroft, 2001; Koustov et al., 2004). The quantitative assessment of this effect is difficult to perform for a large amount of data considered in this study. The other potential factor is deterioration of the quality of radar echo location measurements at very large ranges due to propagation effects. On the magnetometer side, a potential problem at high latitudes is the fact that magnetic perturbations here are weak and often produced by a combined effect of the currents flowing above the magnetometer and distant currents that are often much stronger. It is difficult to estimates the effect. We tested how sensitive are magnetometer-radar disagreements to the intensity of the magnetic perturbations. To accomplish this, we sorted the data obtained in all time sectors and at all magnetic latitudes according to magnetic disturbance, between $5 \mathrm{nT}$ and $200 \mathrm{nT}$. We found that while for low magnetic disturbances $(<20 \mathrm{nT})$, the amount of points of good agreement $\left(\Delta \theta\right.$ within $\left.\pm 45^{\circ}\right)$ is of the order of 40 $45 \%$, for magnetic perturbations in between 100 and $200 \mathrm{nT}$, the amount of such points increases to $\sim 70 \%$. We then can suggest that MEC at very high latitudes can be affected by distant currents and not reflect the real overhead current and convection.

Our comparison showed that the degree of agreement between the MEC direction and ionospheric convection varies with the magnetic latitude and MLT sector. It is expected that a number of factors influence the relationship. For the auroral zone observations, we think that the major factor is spatial conductance variations produced by local precipitation. There might be other effects involved. For example, Liang (2004) studied SuperDARN convection vortices and associated vortices in equivalent currents and showed that the SuperDARN clockwise convection vortices seen on duskside almost always had their counterparts in MEC. The MEC vortices were often shifted equatorward and slightly to the west with respect to the SuperDARN vortices, somewhat similar to observations by Lyatsky et al. (1999). For the clockwise MEC dawnside vortices, the SuperDARN radars showed not 
a pure vortex but rather a round vortical flow with the center of curvature at very high latitudes. Liang (2004) suggested that the poor relationship of the dawn-side vortices in convection and equivalent currents is resulted from modification of the ionospheric Pedersen conductance (but not Hall conductance) in the areas of intense field-aligned currents flowing into the ionosphere and supporting the vortices.

Presented in this study results can be summarized as follows:

1. Statistical comparison of the magnetic equivalent convection direction and ionospheric convection as seen by the SuperDARN radars for more than 39000 joint measurements over three moderately disturbed days showed that the magnetometers give reasonable estimates of the convection direction in more than $55 \%$ of cases with only slightly better agreement for the sunlit conditions. The magnetometer estimate was classified as reasonable one if the difference of the magnetic equivalent convection with the ionospheric convection was less than $45^{\circ}$. Magnetometers give the best (worst) estimates of the convection direction for the sunlit (dark) ionosphere. There is no clear seasonal effect. For the ionosphere in the transition region between the sunlit and dark ionospheres, the magnetometer estimates of the convection direction are of intermediate quality as compared to observations in the sunlit and dark ionospheres.

2. Consideration of 75000 joint magnetometer-radar measurements over five moderately disturbed days and sorting the data according to an MLT sector and magnetic latitude showed that the best agreement of magnetic equivalent convection direction and ionospheric convection occurs in the dusk sector. This observation is interpreted as a consequence of strong electric field control of the magnetic perturbations in the dusk sector as opposed to the midnight-dawn sector where magnetic perturbations are stronger affected by gradients of the ionospheric conductance. The worst agreement between the magnetic equivalent convection and SuperDARN-inferred convection was observed for data gathered at very high latitudes for all MLT sectors but the dusk/evening sector. Significant differences were also observed at very low magnetic latitudes on the dayside, between 09:00 and 15:00 MLT. The width of all considered distributions varies between $50^{\circ}$ and $70^{\circ}$ with only one obvious trend for the distributions to be broader in the midnight/early morning sector.

3. There are a number of reasons for observed discrepancies. One of these is variations in the ionospheric conductance associated with the solar illumination and local precipitations. The data point to no significant effect of the regular solar illumination whereas local conductance variations are clearly important. Another potentially important effect is modification of the high- latitude ionospheric conductance in the areas of fieldaligned currents flowing into the ionosphere. For magnetic observations at high magnetic latitudes, the effect of distant currents can be important for estimates of the ionospheric convection direction.

Acknowledgements. We acknowledge the support of all national funding agencies that made possible continuous operation of the SuperDARN radars whose data were used in this study. We thank organizations and individuals who provided magnetometer data for this study: University of Alaska (GIMA project); Canadian Space Agency (CANOPUS project); Augsburg College, M. J. Engebretson and Boston University, J. Hughes (MACCS project); Geophysical Survey of Canada, R. Coles (Natural Resources Canada magnetometers), Finnish Meteorological Institute (IMAGE network) and Lancaster University (SUMNET project). This work has been supported by NSERC (Canada) grant to A. V. Koustov.

Topical Editor M. Pinnock thanks two referees for their help in evaluating this paper.

\section{References}

Benkevitch, L. and Lyatsky, W.: Detached vortices in equivalent ionospheric currents in the winter dayside ionosphere, Geophys. Res. Lett., 27, 1375-1378, 2000.

Benkevitch, L. V.: Effects of ionospheric conductance in highlatitude phenomena, PhD Thesis, U of Saskatchewan, Saskatoon, SK, Canada, 2006.

Brekke, A., Doupnik, J. R., and Banks, P. M.: Incoherent scatter measurements of $\mathrm{E}$ region conductivities and currents in the auroral zone, J. Geophys. Res., 79, 3773-3790, 1974.

Fukushima, N.: Generalized theorem of no ground magnetic effect of vertical current connected with Pedersen currents in the uniform conductivity ionosphere, Rep. Ionos. Space Res. Jap., 30, 35-40, 1976.

Greenwald, R. A., Baker, K. B., Dudeney, J. R., Pinnock, M., Jones, T. B., Thomas, E. C., Villain, J.-P., Cerisier, J.-C., Senior, C., Hanuise, C., Hunsuker, R. D., Sofko, G., Koehler, J., Nielsen, E., Pellinen, R., Walker, A. D. M., Sato, N., and Yamagishi, H.: DARN/SuperDARN: A global view of the dynamics of highlatitude convection, Space Sci. Rev., 71, 763-796, 1995.

Huang, C. S., Murr, D., Sofko, G. J., and Moretto, T.: Ionospheric convection response to changes of interplanetary magnetic field $\mathrm{Bz}$ component during strong By component, J. Geophys. Res., 105, 5231-5243, 2000.

Kamide, Y.: Electrodynamic processes in the Earth's ionosphere and magnetosphere, Kyoto Sangyo Press, Kyoto, Japan, 1988.

Kamide, Y. and Kokubun, S.: Two-component auroral electrojet: Importance for substorm studies, J. Geophys. Res., 101, $13027-$ $12046,1996$.

Kivelson, M. and Russell, C. T.: Introduction to Space Physics, Cambridge University Press, 1995.

Koustov, A. V., Danskin, D. W., Makarevitch, R. A., and Gorin, J. D.: On the relationship between the velocity of E-region HF echoes and ExB plasma drift, Ann. Geophys., 23, 371-378, 2005 ,

http://www.ann-geophys.net/23/371/2005/. 
Lacroix, P. J. and Moorcroft, D. R.: Ion acoustic HF radar echoes at high latitudes and far ranges, J. Geophys. Res., 106, 29091$29103,2001$.

Liang, J.: Joint study of high-latitude ionosphere by SuperDARN, magnetic, and optical observations, PhD Thesis, U of Saskatchewan, Saskatoon, SK, Canada, 2004.

Lyatsky, W. B. and Maltsev, Y. P.: Influence of solar terminator on electric field and field-aligned currents, Kosmicheskie Issledovaniya (in Russian), 20, 304-308, 1982.

Lyatsky, W. B., Koustov, A. V., Sofko, G. J., Jacobsen, B., Andre, D., and Cogger, L. L.: Ionospheric convection and equivalent ionospheric currents in the dayside high-latitude ionosphere, J. Geophys. Res., 104, 22 525-22 534, 1999.

Benkevitch, L., Lyatsky, W., and Cogger, L. L.: Field-aligned currents between conjugate hemispheres, J. Geophys. Res., 105, 27 727-27 738, 2000.
Milan, S. E., Yeoman, T. K., Lester, M., Thomas, E. C., and Jones, T. B.: Initial backscatter occurrence statistics from the CUTLASS HF radars, Ann. Geophys., 15, 703-718, 1997, http://www.ann-geophys.net/15/703/1997/.

Richmond, A. D. and Kamide, Y.: Mapping electrodynamic features of the high-latitude ionosphere from localized observations: Technique, J. Geophys. Res., 93, 5741-5759, 1988.

Ruohoniemi, J. M. and Baker, K. B.: Large-scale imaging of highlatitude convection with Super Dual Auroral Radar Network HF radar observations, J. Geophys. Res., 103, 20 797-20 811, 1998.

Zhu, L., Gifford, P., Sojka, J. J., and Schunk, R. W.: Model study of ground magnetic signatures of traveling convection vortices, J. Geophys. Res., 102, 7449-7459, 1997. 